ARTICLE

\title{
$\beta 1$-adrenergic receptors mediate plasma acyl-ghrelin elevation and depressive-like behavior induced by chronic psychosocial
}

\section{stress}

\author{
Deepali Gupta ${ }^{1}$, Jen-Chieh Chuang ${ }^{1}$, Bharath K. Mani ${ }^{1}$, Kripa Shankar ${ }^{1}$, Juan A. Rodriguez $\mathbb{D}^{1}$, Sherri Osborne-Lawrence ${ }^{1}$, \\ Nathan P. Metzger ${ }^{1}$ and Jeffrey M. Zigman ${ }^{1,2,3}$
}

The ghrelin system is a key component of the mood and metabolic responses to chronic psychosocial stress. For example, circulating acyl-ghrelin rises in several rodent and human stress models, administered acyl-ghrelin induces antidepressant-like behavioral responses in mice, and mice with deleted ghrelin receptors (GHSRs) exhibit exaggerated depressive-like behaviors, changed eating behaviors, and altered metabolism in response to chronic stress. However, the mechanisms mediating stressinduced rises in ghrelin are unknown and ghrelin's antidepressant-like efficacy in the setting of chronic stress is incompletely characterized. Here, we used a pharmacological approach in combination with a 10-day chronic social defeat stress (CSDS) model in male mice to investigate whether the sympathoadrenal system is involved in the ghrelin response to stress. We also examined the antidepressant-like efficacy of administered ghrelin and the synthetic GHSR agonist GHRP-2 during and/or after CSDS. We found that administration of the $\beta 1$-adrenergic receptor ( $\beta 1 \mathrm{AR}$ ) blocker atenolol during CSDS blunts the elevation of plasma acyl-ghrelin and exaggerates depressive-like behavior. Neither acute injection of acyl-ghrelin directly following CSDS nor its chronic administration during or after CSDS nor chronic delivery of GHRP-2 during and after CSDS improved stress-induced depressive-like behavior. Thus, $\beta 1$ ARs drive the acyl-ghrelin response to CSDS, but supplementing the natural increases in acyl-ghrelin with exogenous acyl-ghrelin or GHSR agonist does not further enhance the antidepressant-like actions of the endogenous ghrelin system in the setting of CSDS.

Neuropsychopharmacology (2019) 44:1319-1327; https://doi.org/10.1038/s41386-019-0334-7

\section{INTRODUCTION}

Chronic stress induces changes in mood, feeding, and metabolism and contributes to many psychiatric and metabolic disorders [1-3]. To develop more effective interventions for these disorders, a thorough understanding of the mechanisms underlying chronic stress is needed. To that end, we have been investigating the role of the hormone ghrelin using a 10-d chronic social defeat stress (CSDS) mouse model of chronic psychosocial stress.

Ghrelin is a peptide secreted predominantly from the stomach [4]. Ghrelin is present in circulation in an unacylated form and as acyl-ghrelin, the latter being responsible for ghrelin's well-known orexigenic actions [4] and its antidepressant-like actions (see below). Acyl-ghrelin works by engaging the only known ghrelin receptor, growth hormone secretagogue receptor (GHSR), which is expressed in select peripheral tissues and several brain regions, including many that regulate mood, reward, and feeding [4].

Elevations in plasma ghrelin, ghrelin gene expression, stomach ghrelin protein content, and/or ghrelin cell numbers occur in several stress models, with rises in plasma acyl-ghrelin occurring most consistently with chronic stress as opposed to acute stress $[5,6]$. In rodents, repeated tail pinch stress, maternal immobilization stress during lactation, repeated restraint stress, and chronic housing in water-filled cages raise gastric ghrelin gene expression [7-10]. Also, repeated restraint stress, chronic isolation stress, chronic water stress, and 3 wks of CSDS raise plasma acyl-ghrelin $[6,9,11,12]$. Notably, the elevated plasma acyl-ghrelin in rats exposed to 2 wks of $4 \mathrm{~h}$-per-day immobilization stress persists for at least 130 days [6]. Long-lasting, chronic stressinduced rises in plasma acyl-ghrelin also occur in traumatized adolescents; those traumatized by terror attacks on average 4.5 years earlier exhibit 2-fold higher plasma acyl-ghrelin than non-traumatized controls [13]. Likewise, using the same 10-d CSDS mouse protocol of the current study, we previously demonstrated about 2-3-fold rises in plasma acyl-ghrelin, which persisted at least 30 days [14-16].

Stress-induced changes in the ghrelin system impact eating, metabolism, fear learning, anxiety-related behavior, and gastric motility $[2,3,5,6,13,17-20]$. For instance, as it regards eating and metabolism, wild-type mice exposed to the 10-d CSDS protocol exhibit hyperphagia and conditioned place preference for high fat diet rewards, whereas CSDS-exposed transgenic mice lacking GHSR expression (GHSR-null mice) do not [14, 15]. Similarly,

\footnotetext{
${ }^{1}$ Department of Internal Medicine, Division of Hypothalamic Research, UT Southwestern Medical Center, 5323 Harry Hines Blvd., MC9077, Dallas, TX 75390-9077, USA; ${ }^{2}$ Department of Internal Medicine, Division of Endocrinology, UT Southwestern Medical Center, Dallas, TX, USA and ${ }^{3}$ Department of Psychiatry, UT Southwestern Medical Center, Dallas, TX, USA

Correspondence: Jeffrey M. Zigman (jeffrey.zigman@utsouthwestern.edu)

These authors contributed equally: Deepali Gupta, Jen-Chieh Chuang
}

Received: 16 August 2018 Revised: 6 December 2018 Accepted: 4 February 2019

Published online: 8 February 2019 
following 3 wks of CSDS, a different line of mice with genetic GHSR deletion as well as wild-type mice receiving chronic GHSR antagonist infusion exhibited attenuated stress-induced weight gain and hyperphagia [12].

Here we focus on depressive-like behavior. Both human and rodent studies report antidepressant-like effects for various components of the ghrelin system, albeit the data for humans is limited [5]. In humans, the Leu72Met ghrelin gene polymorphism occurs with more frequency in subjects with major depressive disorder than in controls [21]. Also, following a short-term course of acyl-ghrelin (four successive hourly injections), there is a marginally significant reduction in depressive symptom severity in men with major depressive disorder [22]. In non-stressed mice, acute acyl-ghrelin administration exerts antidepressant-like effects, as assessed using the forced swim and tail suspension tests $[14,23]$. Acute acyl-ghrelin administration also reverses the depression-like phenotype of olfactory bulbectomized mice [23]. While 10-d of mild calorie restriction raises plasma acyl-ghrelin and exerts an antidepressant-like response in the forced swim test, calorie-restricted GHSR-null mice lack this antidepressant-like response [14]. GHSR-null mice also exhibit exaggerated social avoidance following $10 \mathrm{~d}$ of CSDS [14, 15, 24]. This exaggerated depressive-like response of CSDS-exposed GHSR-null mice is associated with a greater reduction in hippocampal neurogenesis than that occurring in CSDS-exposed wild-type mice [24].

A major aim of the current study is to investigate the mechanism for the stress-induced elevation in plasma acylghrelin, focusing on the actions of the sympathoadrenal system, and in particular, $\beta 1$ ARs. Importantly, it has long been appreciated that during exposure to stress, sympathetic nervous system outflow increases, leading to stimulation of the cardiovascular system, increased core body temperature, raised locomotor activity, and the release of adrenal catecholamines [25-27]. This collection of sympathetic nervous system-driven changes is a wellknown component of psychosocial stress, including that modeled by CSDS, and is at the core of the classical "fight or flight" reaction to a perceived or actual stressor [25-27]. Also of note, the sympathetic nervous system has been shown to be an important regulator of ghrelin secretion. Not only does stimulation of sympathetic neurons increase ghrelin release in rodent models [28], but also pharmacologic antagonism of $\beta 1$ ARs and genetic deletion of $\beta 1$ ARs from gastric ghrelin cells markedly attenuate the increase in ghrelin secretion associated with calorie restriction $[29,30]$. We will accomplish this aim by determining the effect of the $\beta$-blocker atenolol on ghrelin secretion stimulated in mice exposed to the 10-d CSDS model. Another aim of the current study is to investigate the antidepressant-like efficacy of administered acyl-ghrelin or GHSR agonist using the 10-d CSDS model.

\section{METHODS AND MATERIALS}

Animals and housing

Male C57BL/6 N mice (8-10 weeks old) and male retired breeder CD1 mice (Charles River Laboratories Inc., Wilmington, MA) were housed in the UT Southwestern Medical Center (UTSW) vivarium in a temperature-controlled environment (lights on: 0600-1800) with ad lib access to water and standard chow (\#7001, HarlanTeklad, Madison, WI). All procedures were approved by the UTSW Institutional Animal Care and Use Committee.

\section{CSDS and Social Interaction Test}

CSDS was performed as described in [31] (except for small changes in the cage dimensions), as we have done previously $[14-16,24,32]$. Each test mouse was housed individually in one half of a $43 \mathrm{~cm}(w) \times 23 \mathrm{~cm}(d) \times 15 \mathrm{~cm}(h)$ plastic cage separated by a perforated plastic divider from a CD1 mouse (screened previously for aggressiveness) in the other half of the cage. At
1600, the plastic divider was removed, allowing a physical encounter. After $5 \mathrm{~min}$, the plastic divider was replaced to again physically separate the mice for the remainder of the $24 \mathrm{~h}$. This was repeated for 10 consecutive days, during which each test mouse was paired with a new CD1 mouse daily. A Social Interaction Test was performed at 0900 on Day 11 (and again on Day 26 , when indicated) by placing the test mouse in a $44 \times$ $44 \times 30 \mathrm{~cm}$ plastic cage with a $10 \times 6 \times 30 \mathrm{~cm}$ wire-mesh cage at one end. The movements of and distance traveled by the test mouse were tracked using Ethovision 5.0 software (Noldus, Leesburg, VA) for $5 \mathrm{~min}$, during the last $2.5 \mathrm{~min}$ of which an unfamiliar CD1 "target" mouse was present within the wire-mesh cage. The time spent in a narrow interaction zone surrounding the wire-mesh cage $(6.5 \mathrm{~cm}$ wide in front; $5.5 \mathrm{~cm}$ wide at each side) during the last $2.5 \mathrm{~min}$ is reported.

\section{Drug administration}

Atenolol (Sigma Aldrich, St. Louis, MO) freshly-prepared daily in $2 \mathrm{mM} \mathrm{HCl}$ (vehicle) was delivered i.p. [10 mg/kg body weight (BW)] twice-daily at 0600 and 1730 during the 10 days of CSDS and at 0600 on Day 11. For acute acyl-ghrelin administration, acyl-ghrelin (Pi Proteomics, Huntsville, AL) dissolved in saline was delivered s.c. ( $2 \mathrm{mg} / \mathrm{kg}$ BW) $45 \mathrm{~min}$ prior to the Social Interaction Test on Day 11. For chronic acyl-ghrelin administration, acyl-ghrelin was delivered via s.c. osmotic pump ( $4 \mathrm{mg} / \mathrm{kg}$ BW/day) either from 2 days prior to CSDS to one day after CSDS or from 2 days after CSDS to 15 days later, as noted. Growth hormone releasing peptide-2 (GHRP-2; Sigma Aldrich) dissolved in saline was delivered s.c. $(400 \mu \mathrm{g} / \mathrm{kg} \mathrm{BW} /$ day; about $10 \mu \mathrm{g} /$ day $)$ via an osmotic pump implanted 2 days prior to CSDS and continued for 28 days. Control mice received equal volumes of saline or vehicle. These doses are based on previous studies [29, 33, 34].

Surgical implantation of osmotic minipumps

Osmotic pumps (Alzet, Cupertino, CA; model 1002) prefilled with saline, $16.7 \mathrm{mg} / \mathrm{mL}$ rat acyl-ghrelin, or $4.0 \mathrm{mg} / \mathrm{mL}$ GHRP-2 were inserted s.c. into the interscapular region of mice under anesthesia. Acyl-ghrelin $(0.25 \mu \mathrm{L} / \mathrm{h})$ and GHRP-2 $(0.11 \mu \mathrm{L} / \mathrm{h})$ were delivered to achieve the noted daily doses.

Determination of plasma acyl-ghrelin

Blood samples were collected by a superficial temporal vein bleed into iced EDTA-coated microtubes containing protease inhibitor $p$-hydroxymercuribenzoic acid (final concentration $1 \mathrm{mM}$ ). The samples were immediately centrifuged at $4{ }^{\circ} \mathrm{C}$, at $1,500 \times g$, for $15 \mathrm{~min}$, and $\mathrm{HCl}$ was added to the supernatant to achieve a final concentration of $0.1 \mathrm{~N}$. Acyl-ghrelin concentrations were determined by ELISA [Millipore-Merck; Burlington, MA (Fig. 1); Cayman Chemicals; Ann Arbor, MI (Fig. 2)] with the help of a PowerWave XS Microplate spectrophotometer and KC4 Junior software (BioTek Instruments, Winooski, VT).

Determination of GHSR mRNA expression

GHSR mRNA expression in the VTA and nucleus accumbens was determined as reported [15]. Notably, while GHSR mRNA expression in the VTA is relatively substantial and likely relevant to the ghrelin system's antidepressant-like effects during CSDS, GHSR expression in the nucleus accumbens is barely detectable and is included here as a negative control [15]. Brains were extracted after live decapitation, placed in cold diethylpyrocarbonate-PBS, and then sectioned into $1-\mathrm{mm}$ coronal slices in a stainless steel mouse brain matrix. Tissue punches corresponding to the regions of interest, as confirmed by comparison to a mouse brain atlas, were excised using blunted 15-g needles. Total RNA was isolated using RNA STAT-60 (Tel-Test Inc., Friendswood, TX). After its concentration was determined with a Thermo Scientific Nanodrop 100 Spectrophotometer, total RNA was treated with RNase-free DNase (Roche Diagnostics Corporation, Indianapolis, 
IN) and reverse-transcribed into CDNA with SuperScript II reagents (Invitrogen, Carlsbad, CA). Quantitative PCR was performed using an Applied Biosystems 7900HT Sequence Detection System and SYBR Green chemistry (Applied Biosystems, Foster City, CA) using previously designed and validated primers [15]. The mRNA levels were evaluated by the comparative threshold cycle $(\Delta \Delta \mathrm{Ct})$ method using 36B4 as the invariant control gene [15]. The data are presented as values normalized to the saline control group.

Statistics

The data are reported as mean \pm S.E.M. Statistical analyses were performed by Student $t$ test or repeated measures analysis of variance (ANOVA) followed by Sidak post-hoc comparison test, using GraphPad Prism 6.0 (La Jolla, CA). Statistical significance was defined as $p<0.05$.

\section{RESULTS}

Effects of atenolol on plasma acyl-ghrelin and behavior in CSDSexposed mice

As $\beta 1 A R$ signaling is essential for the increase in plasma ghrelin associated with caloric restriction [29], we determined the effect of atenolol on plasma acyl-ghrelin in CSDS-exposed mice. We chose atenolol as the $\beta$-blocker for this study because of its high selectivity for $\beta 1 A R$, its relatively high hydrophilicity (which is thought to limit the potential for central side effects), and its prevalent usage as an antihypertensive and chronotropic agent, thus providing translational relevance [35-37]. Furthermore, the effects of atenolol on ghrelin secretion from cultured ghrelin cells and on plasma ghrelin levels have been characterized previously (albeit not in the setting of stress) [29, 30]. Administration of atenolol twice daily during CSDS (Days 1-10) and on the morning of the Day 11 Social Interaction Test lowered plasma acyl-ghrelin by $36.5 \%$ (Fig. 1a, b). Atenolol also exaggerated the depressive-like behavior of these CSDS-exposed mice, as indicated by a decrease in time spent in interaction zone when the target mouse was present (or rather, greater social avoidance; Fig. 1c). Atenolol did not impact body weight change or food intake (Fig. 1d, e). Nor did atenolol impact locomotor activity, as assessed by distance traveled during the Day 11 Social Interaction Test in the absence or presence of the target mouse (Figure S1).

Effects of acute ghrelin injection on depressive-like behavior in CSDS-exposed mice

Next, we tested whether an acute, pharmacologic rise in plasma acyl-ghrelin just prior to the Social Interaction Test could limit CSDS-induced social avoidance. A single dose of acyl-ghrelin s.c. $45 \mathrm{~min}$ prior to the Day 11 Social Interaction Test raised plasma

A

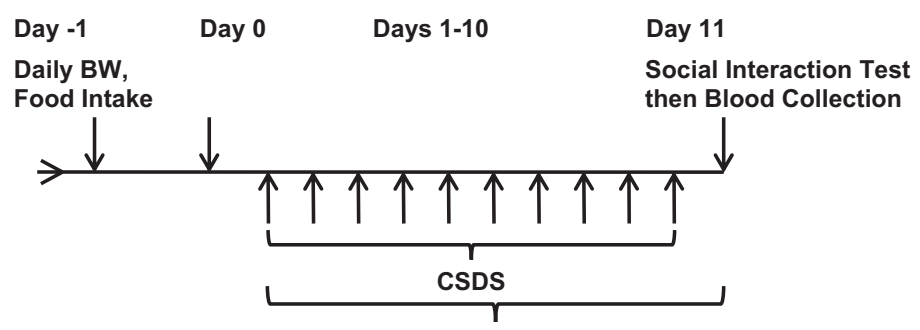

Chronic Vehicle vs. Atenolol Injection

(10 mg/kg BW/twice daily i.p.)
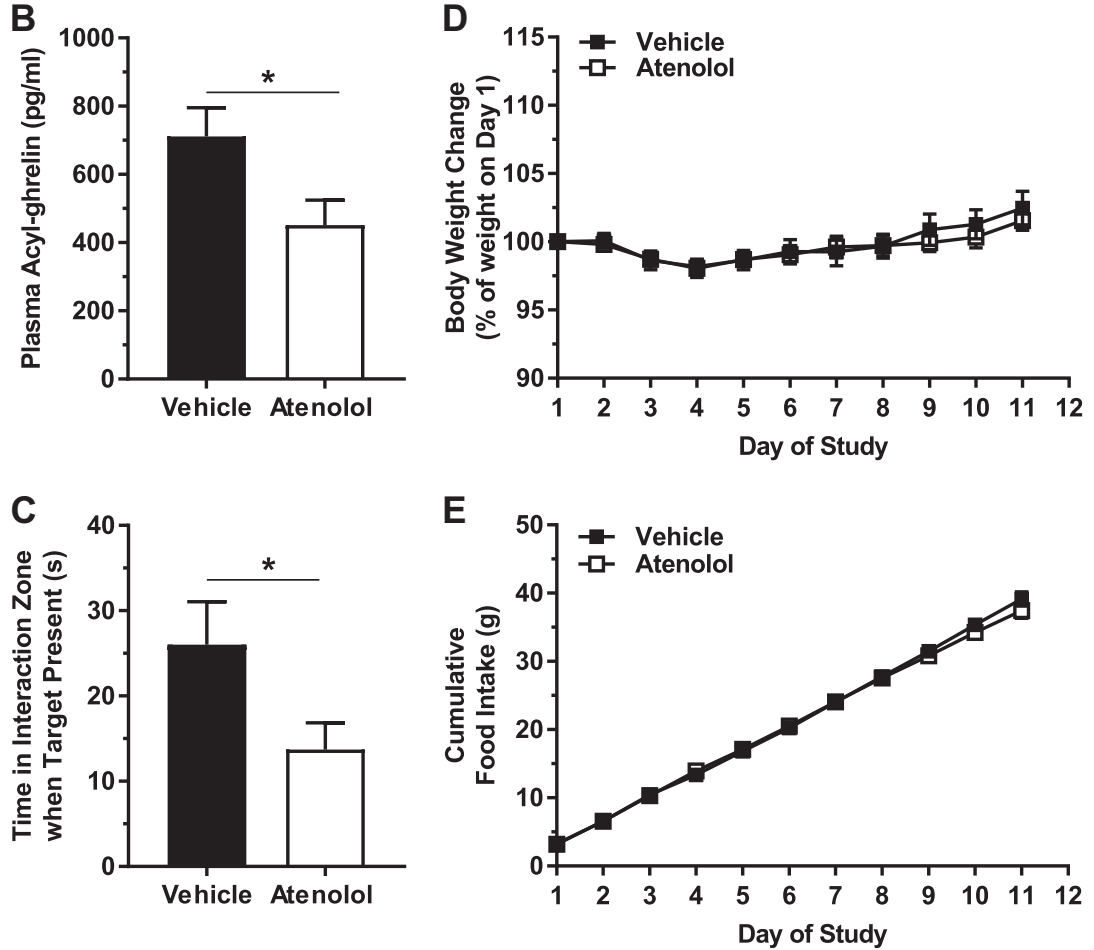

Fig. 1 Effects of chronic atenolol administered during CSDS. a Schematic diagram showing the timeline of the experiment. b Plasma acyl-ghrelin. c Social interaction behavior. d Body weight change and (e) cumulative food intake over the course of the study. $n=18$ per group. ${ }^{*} p<0.05$ 
A
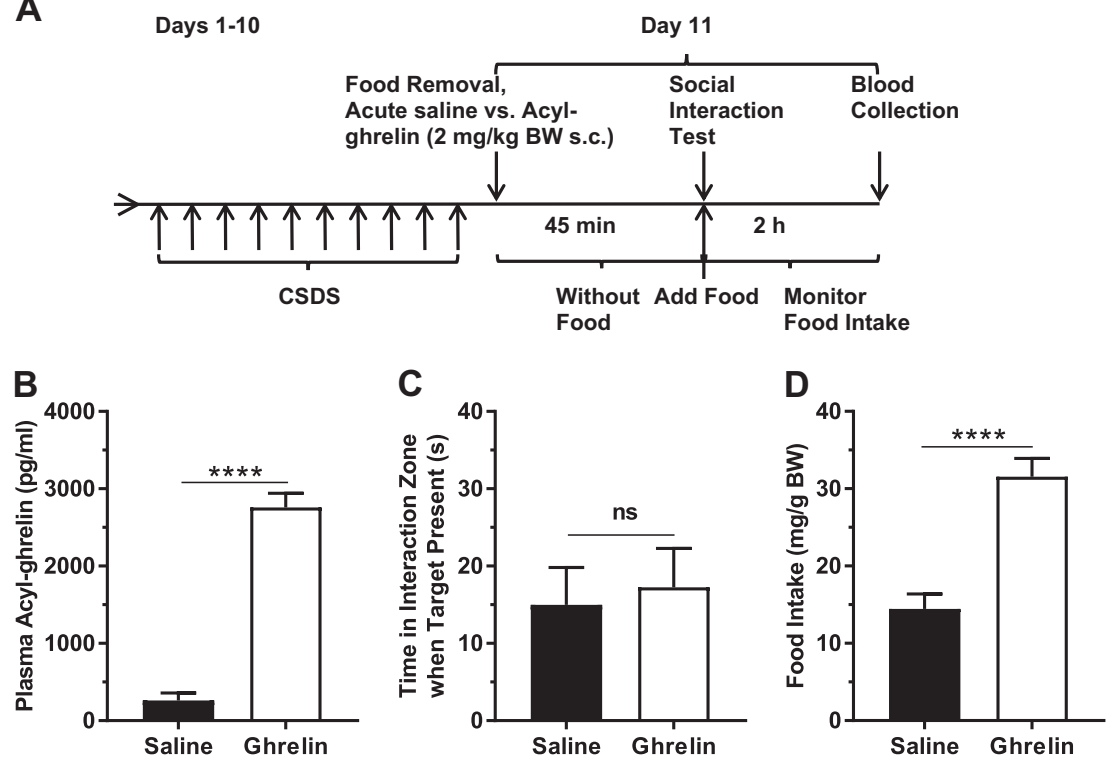

Fig. 2 Effects of acute ghrelin administered to CSDS-exposed mice. a Schematic diagram showing the timeline of the experiment. $\mathbf{b}$ Plasma acyl-ghrelin. c Social interaction behavior. d Two-hour food intake following the Social Interaction Test. $n=15$ per group. ${ }^{* * * *} p<0.0001$, ns no significant difference

acyl-ghrelin (Fig. 2a, b). Social avoidance remained unchanged (Fig. 2c). Consistent with the well-described orexigenic effects of administered acyl-ghrelin [4], $2 \mathrm{~h}$ food intake following the Social Interaction Test increased (Fig. 2d).

Effects of chronic ghrelin infusion on depressive-like behavior in CSDS-exposed mice

As the therapeutic efficacy of most antidepressant agents requires several weeks to become apparent [31], we next examined the behavioral effects of chronic acyl-ghrelin administration either during or after CSDS. Acyl-ghrelin infusion from two days prior to CSDS until after the Day 11 Social Interaction Test did not affect social avoidance (Fig. 3a, b). However, it did enhance body weight increase [alike previously reported [33]] and cumulative food intake (Fig. 3c, d). A second chronic acyl-ghrelin infusion was performed over 15 days beginning two days after CSDS -- one day after an initial Social Interaction Test, with a second Social Interaction Test on the final day (Fig. 4a). As expected, the social avoidance behaviors of the groups following CSDS but prior to randomization to either acyl-ghrelin or saline infusion were similar (Fig. 4b). Chronic acyl-ghrelin after CSDS did not alter social avoidance (Fig. 4c). Body weight gain and cumulative food intake both increased (Fig. 4d, e). Chronic acyl-ghrelin infusion also reduced GHSR mRNA levels within the VTA. The barely-detectable nucleus accumbens GHSR mRNA expression was unaffected (Fig. 4f).

Effects of chronic GHRP-2 infusion on depressive-like behavior in CSDS-exposed mice

Next, we examined the effect of the GHSR agonist GHRP-2 on depressive-like behavior of CSDS-exposed mice. GHRP-2 has a longer-half-life than acyl-ghrelin, with many similar actions [34]. The 28-day infusion protocol with GHRP-2 was chosen not only to extend the period of administered GHSR agonist, but also to provide a GHSR agonist with more stability than acyl-ghrelin [34]. Chronic GHRP-2 infusion did not influence social avoidance behavior of mice when assessed on either Day 11 or Day 26 (Fig. 5b, c). Unlike the effects of an 18-d course of daily s.c. GHRP-2 administration to increase body weight and food intake in mice [34], the chronic GHRP-2 protocol used here did not impact body weight gain or cumulative food intake (Fig. $5 d$, e).

\section{DISCUSSION}

The current study was designed to further characterize the ghrelin system's response to chronic psychosocial stress, including both the mechanism driving the stress-induced elevation in plasma acyl-ghrelin and the antidepressant-like efficacy of acylghrelin in the setting of chronic stress. We demonstrate for the first time that administration of atenolol during CSDS lowers plasma acyl-ghrelin. When viewed alongside previous studies showing higher plasma acyl-ghrelin in CSDS-exposed mice than in non-CSDS-exposed controls [14-16, 24], these new data suggest that the normal CSDS-induced increase in plasma acylghrelin - and perhaps increases in plasma acyl-ghrelin associated with other stress models - requires intact $\beta 1 A R$ signaling. Furthermore, we demonstrate that atenolol amplifies depressive-like behavior, suggesting that intact $\beta 1 A R$ signaling defends against an exaggerated depressive-like response to CSDS. We also used four different protocols to boost GHSR signaling pharmacologically during and/or after CSDS in an attempt to enhance the endogenous antidepressant-like actions of the ghrelin system, however none was effective. Thus, supplementing the natural increases in plasma acyl-ghrelin with exogenous acyl-ghrelin or GHSR agonist does not enhance the antidepressant-like actions of the endogenous ghrelin system in the setting of CSDS.

Atenolol-induced lowering of plasma acyl-ghrelin in the setting of CSDS was not unexpected. Indeed, a sympathoadrenal-driven "fight or flight" response is a well-known component of psychosocial stress, including that modeled by $\operatorname{CSDS}[26,27]$. Additionally, sympathetic outflow acting via $\beta 1 A R s$ localized to ghrelin cells is an integral mechanism regulating basal ghrelin release and its stimulated release in response to caloric restriction. Supporting this assertion, ghrelin secretion increases when adrenergic agents are infused into the gastric submucosa [38]. Atenolol blocks fasting-induced increases in plasma ghrelin [30]. Norepinephrine, epinephrine, and/or the $\beta A R$ agonist isoproterenol stimulate ghrelin secretion from ghrelinoma cell lines and primary cultures of gastric cells; this is blocked by atenolol [28-30, $39-43]$. Moreover, $\beta 1 A R$ is the most highly expressed and one of the most highly enriched $G$ protein-coupled receptors within gastric ghrelin cells (whereas by comparison, $\beta 2 A R$ is several thousand-fold lower in expression and $\beta 3 A R$ expression is barely 
A
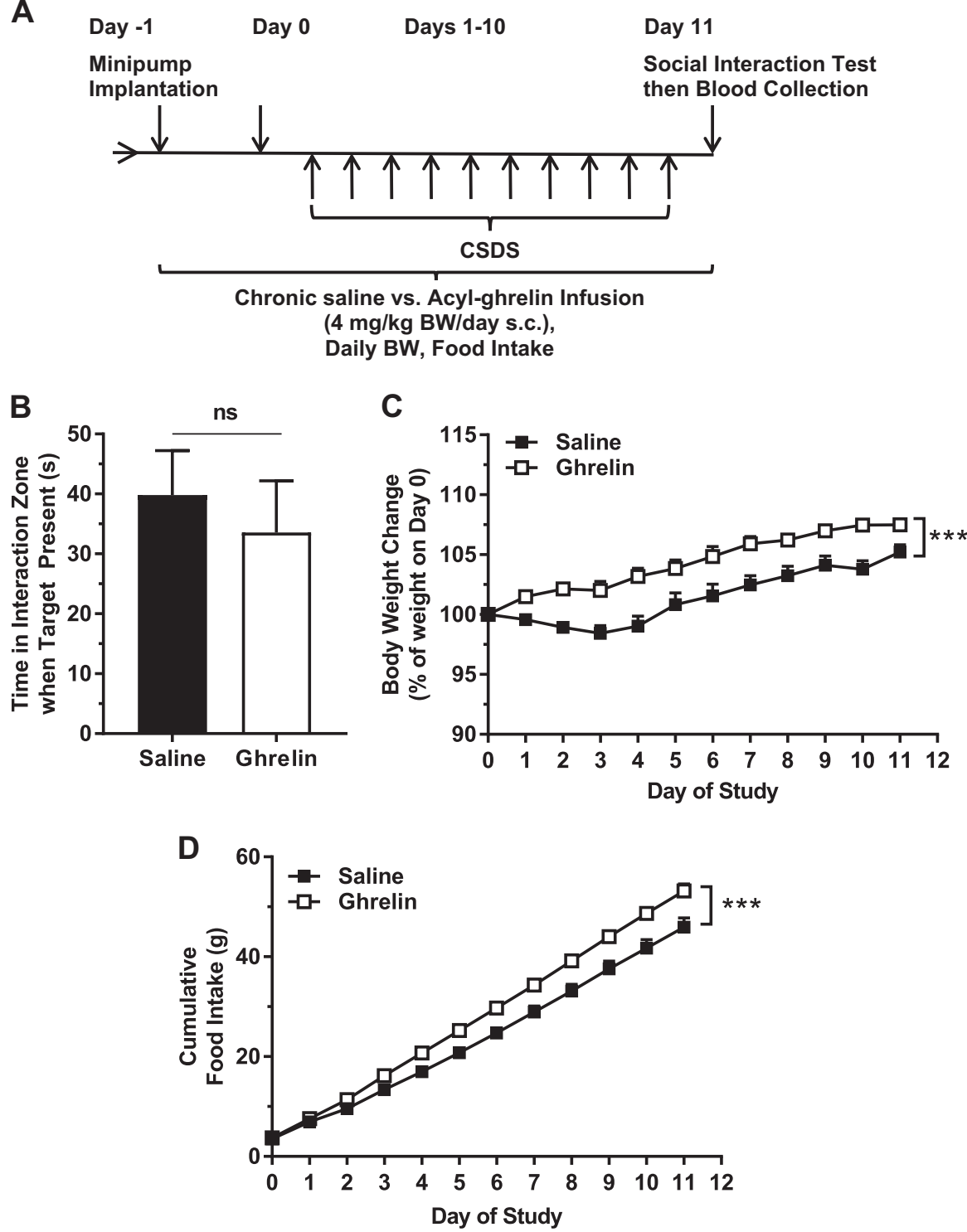

Fig. 3 Effects of chronic ghrelin administered during CSDS. a Schematic diagram showing the timeline of the experiment. b Social interaction behavior. c Body weight change and (d) cumulative food intake over the course of the study. $n=15$ per group. ${ }^{* * *} p<0.005$, ns no significant difference

detectable), and genetic deletion of $\beta 1$ ARs in a ghrelin cellselective manner markedly blunts caloric restriction-induced rises in plasma acyl-ghrelin [29, 30, 43, 44]. The high gastric ghrelin cell $\beta 1$ AR expression, coupled with previous reports identifying gastric ghrelin cells as the predominant source of circulating ghrelin [45] and atenolol as a $\beta 1$ AR-selective $\beta$-blocker with a hydrophilic nature that restricts its actions to the periphery, suggest that the current finding of atenolol-induced lowering of plasma ghrelin in the setting of CSDS is mediated via blocking $\beta 1$ ARs expressed by the gastric ghrelin cells. Also, although not a $\beta$-blocker, the tyrosine hydroxylase inhibitor alpha-methyl-paratyrosine, which causes transient catecholamine depletion, lowers total plasma ghrelin when administered to human subjects; these reduced total ghrelin levels negatively correlate with depressive symptoms [46].

Notably, it is likely that increased sympathetic nervous system outflow is responsible for the stress-induced rise in plasma acylghrelin as opposed to catecholaminergic output from the adrenal glands. As such, electrical stimulation of postganglionic sympathetic axons projecting from the celiac ganglion raises plasma total ghrelin in rats [28]. So does chemical sympathetic nerve activation with i.v. tyramine - an agent without direct effects on the adrenal medulla - whereas i.v. epinephrine infusion to simulate a humoral adrenal-derived epinephrine response, does not [28]. Also, reserpine, which depletes adrenergic neurotransmitters from sympathetic neurons but does not affect adrenal catecholamines, blocks fasting-induced plasma acylghrelin elevation in mice [30]. Of interest, a well-known side effect of reserpine when used as an antihypertensive agent is worsening of depressive symptoms [47]. Additionally, in rats, the chronic immobilization stress-induced elevation in plasma acyl-ghrelin occurs even after surgical removal of the adrenal glands [6].

The effect of atenolol treatment during CSDS to exaggerate depressive-like behavior also warrants discussion. To our knowledge, the current study is one of only a small number of preclinical studies to investigate the effects of $\beta$-blockers on depressive-like behaviors or in the setting of chronic stress. Previously, we demonstrated that daily administration of a selective $\beta 3 A R$ antagonist during the 10- $d$ CSDS protocol worsens social avoidance, similar to the effect of the $\beta 1 A R$ antagonist 
A

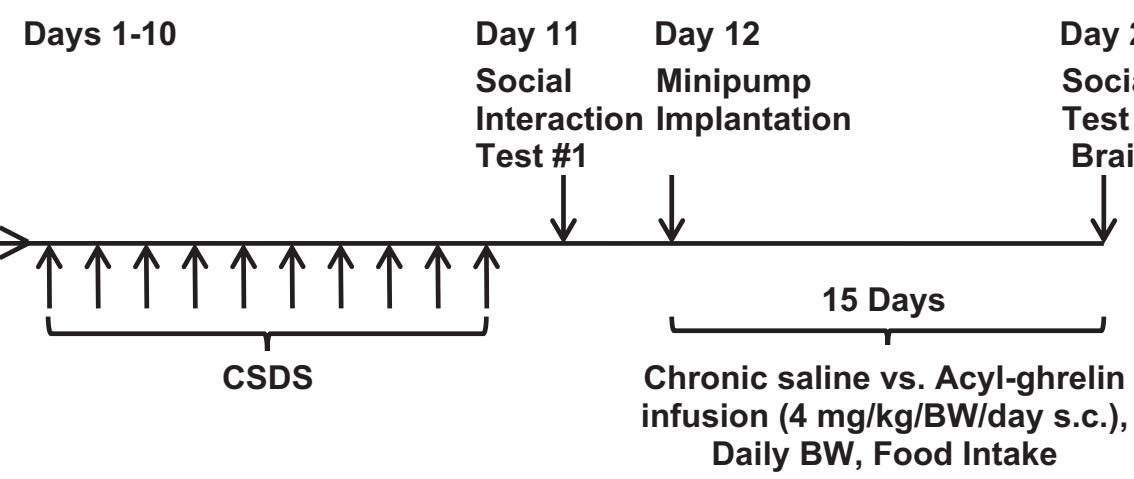

B

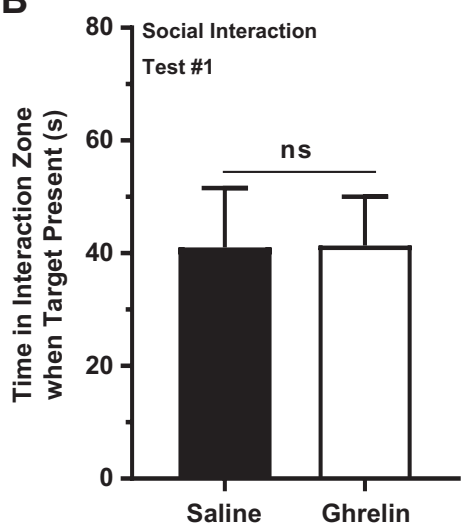

C

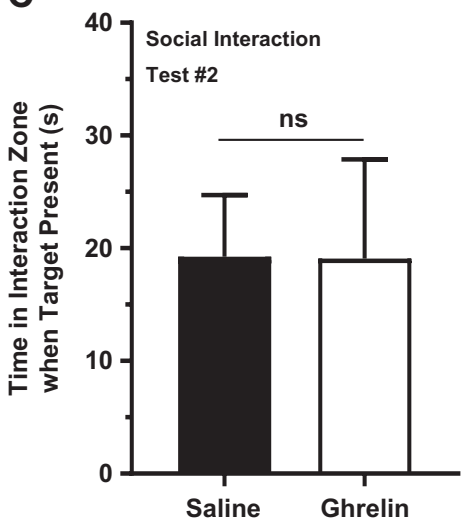

$\mathbf{F}$

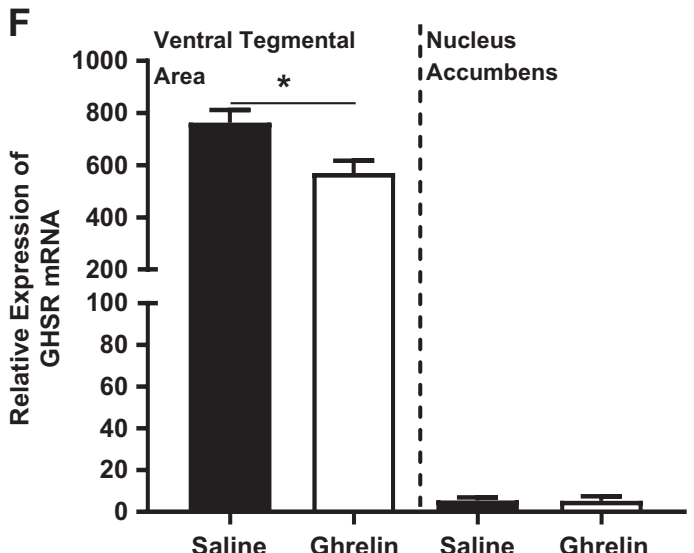

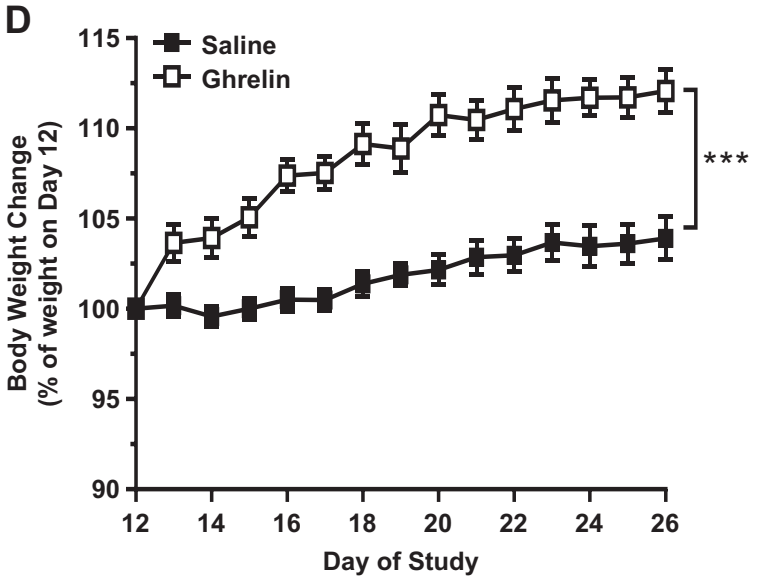

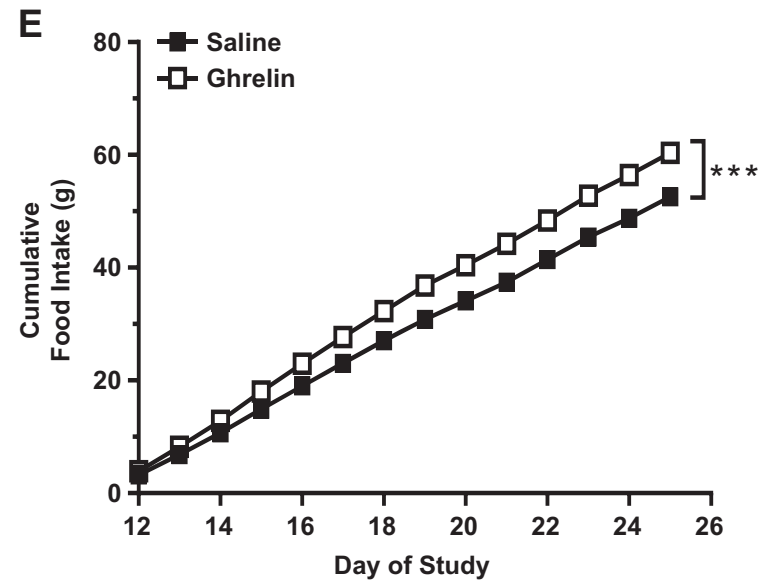

Fig. 4 Effects of chronic ghrelin administered after CSDS. a Schematic diagram showing the timeline of the experiment. b Social interaction behavior in CSDS-exposed mice prior to chronic infusion and (c) after chronic infusion. $\mathbf{d}$ Body weight change and (e) cumulative food intake over the course of the infusion. f GHSR mRNA expression in the VTA and nucleus accumbens after the infusion. $n=6-12$ per group. ${ }^{*} p<0.05$, ${ }^{* * *} p<0.005$, ns - no significant difference

atenolol observed here [32]. This finding correlates with an earlier study in which a selective $\beta 3 A R$ agonist produced antidepressantlike effects in rodent models such as the forced swim and chronic mild stress tests [48]. Thus, these results collectively suggest that both signaling through $\beta 1 \mathrm{ARs}$ - perhaps, mediated at least in part by stimulation of acyl-ghrelin release-and signaling through $\beta 3 A R s$ serve as adaptive responses that help minimize chronic social stress-induced depressive-like behavior.

However, not all studies necessarily support such a conclusion. Although a forced swim test study in non-stressed mice demonstrated that atenolol blocks the antidepressant-like effects of the gut peptide GLP-2, atenolol did not on its own induce depressive-like responses [49]. Oppositely, in a rat forced swim study, atenolol yielded antidepressant-like effects [50]. Other forced swim test mouse studies demonstrated no antidepressant-like effect for the lipophilic, non-selective $\beta A R$ antagonist propranolol, while following several bouts of social defeat stress, a short course of propranolol reversed depressive-like behavior when assessed after re-exposure to stress [51, 52].

Much better represented in the literature are studies examining the potential link between $\beta$-blockers and depression in humans. Several case reports and case series associating $\beta$-blockers (particularly, those with high lipophilicity) with an increased risk for depressive symptoms exist [53-55]. Other studies, though, including a 2002 meta-analysis of 15 randomized-controlled trials with over 35,000 patients treated with $\beta$-blockers, are not supportive of that association [54]. The meta-analysis concluded 
A
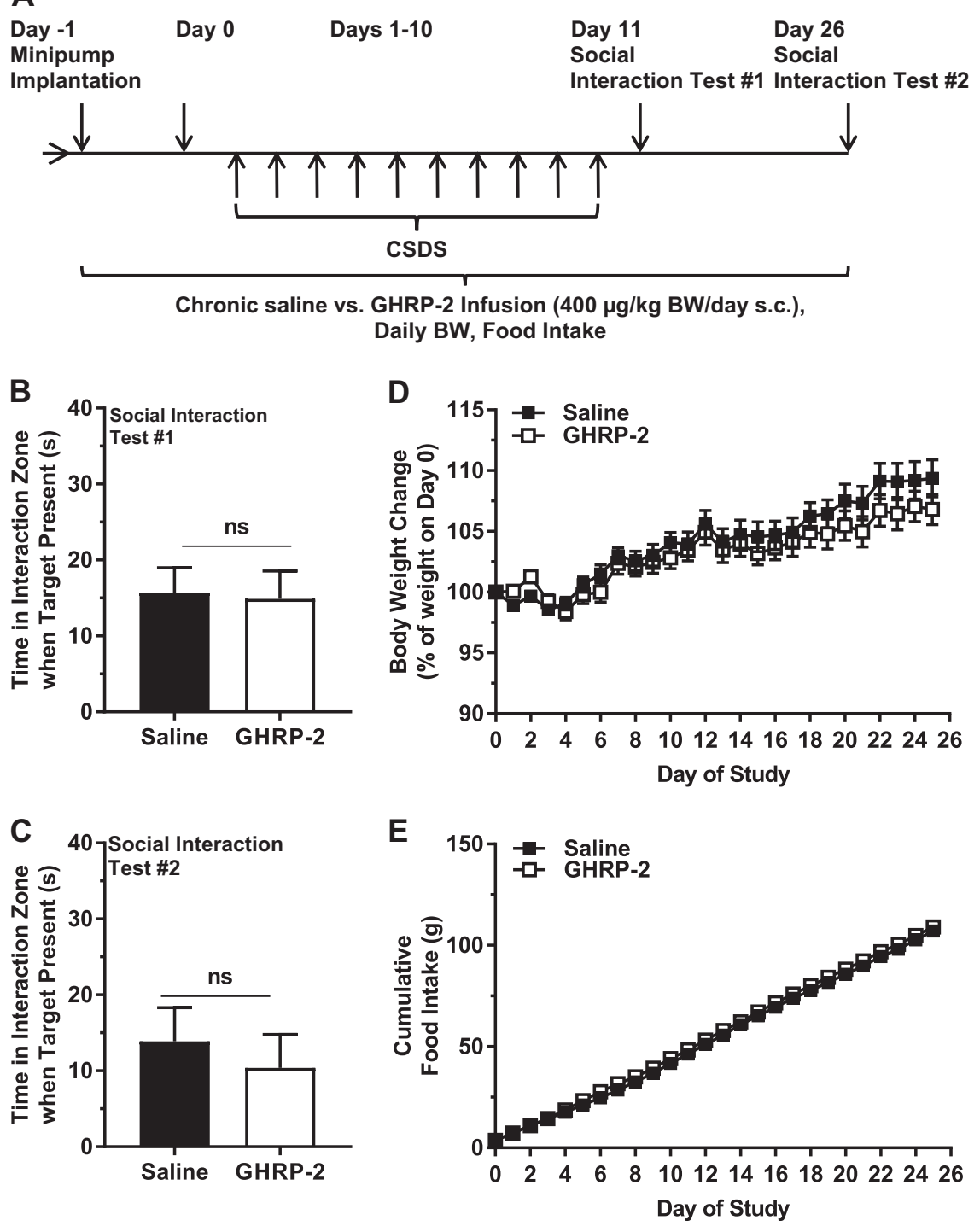

Fig. 5 Effects of chronic GHRP-2 administered during and after CSDS. a Schematic diagram showing the timeline of the experiment. b Social interaction behavior as assessed on Day 11 and (c) Day 26. d Body weight change and (e) cumulative food intake over the course of the study. $n=15$ per group. ns - no significant difference

that $\beta$-blockers did not increase risk of reported depression symptoms, although it has been criticized for assessing fatigue and sexual dysfunction, which were slightly increased, separately from depressive symptoms $[53,54,56]$. A subsequent prospective trial of 1152 hypertensive subjects allocated to either atenololbased or verapamil-based strategies found that among those without pre-existing depression, patients in the atenolol group were more likely to report worsening of depressive-symptoms whereas depressive symptoms improved in those with preexisting depression regardless of the treatment group [57].

It is worth noting that we previously demonstrated that atenolol treatment or ghrelin cell-selective $\beta 1 \mathrm{AR}$ deletion markedly attenuated the rise in plasma ghrelin normally induced by an overnight fast or by exposure to a chronic caloric restriction paradigm $[29,30]$. Importantly, we also showed in those studies that atenolol treatment or ghrelin cell-selective $\beta 1 A R$ deletion lowered "basal" plasma ghrelin levels in ad lib-fed mice [29]. Given that the ad lib-fed mice in those previous studies were not submitted to stress yet still exhibited decreases in plasma ghrelin, we would predict that atenolol would have a similar effect in an unstressed (non-CSDS-exposed) control group if such a group were added to the current study. In other words, the effect of atenolol to block ghrelin release is not restricted to settings that normally stimulate ghrelin secretion (such as caloric restriction and psychosocial stress). Instead, although we did not include a non-CSDS-exposed control group in the current study, our previous studies using ad lib-fed, unstressed mice suggest that atenolol also restricts what is presumed to be basal ghrelin release into the plasma [29]. Thus, $\beta 1$ AR signaling regulates acyl-ghrelin levels in both caloric restricted and ad lib-fed conditions. The current results coupled with our previous work [29] suggest that $\beta 1 A R$ signaling also regulates acyl-ghrelin levels in both stressed and unstressed settings. Therefore, the current results can most conservatively be interpreted as demonstrating that $\beta 1 A R$ signaling permits CSDS-induced plasma ghrelin elevation. Notwithstanding the challenges that would be faced in dissecting $\beta 1 A R^{\prime} s$ effects on basal ghrelin release vs. those on caloric restriction-induced or stress-induced ghrelin secretion, that plasma ghrelin is reduced by atenolol in both extremes of food availability (ad lib-fed condition and caloric restriction) and in both extremes of stress exposure (stressed and unstressed) does not 
preclude important functions for $\beta 1 A R$ in stress-induced ghrelin secretion or depressive-like behaviors induced by stress.

As a final discussion topic, the lack of an antidepressant-like effect of administered acyl-ghrelin or GHRP-2 in the setting of CSDS is curious. Given previous findings of CSDS-induced plasma acyl-ghrelin elevations, antidepressant-like efficacy for acutely administered acyl-ghrelin in the forced swim test, and worsened social avoidance of GHSR-null mice following CSDS, we had predicted that administered acyl-ghrelin or GHRP-2 would improve social interaction. Also, such would be predicted in light of the actions in rats of a 2-wk daily i.c.v. course of ghrelin or the GHSR agonist GHRP-6 to reverse depressive-like behaviors induced by a 6wk mouse chronic unpredictable mild stress protocol [58].

One relatively straightforward speculation for the absent antidepressant-like efficacy of administered acyl-ghrelin or GHRP-2 is that it reflects lack of antidepressant-like efficacy for ghrelin (or GHSR agonist) in the setting of CSDS. Instead, it could be that the exaggerated depressive-like behavior exhibited by GHSR-null mice is due not to (or not only to) loss of acyl-ghrelin action, but instead to (or additionally to) loss of GHSR's presumed high ghrelin-independent, constitutive activity or interruption of potentially important signaling through other $\mathrm{G}$ protein-coupled receptors, such as serotonin and dopamine receptors, with which GHSRs are known to heterodimerize [3, 4]. Alternatively, the elevated levels of endogenous ghrelin that occur naturally in response to stress may lead to a state of GHSR binding saturation beyond which the administration of more acyl-ghrelin (or GHRP-2) produces little or no additional benefit.

The absent behavioral effect of administered acyl-ghrelin or GHRP-2 also might be related to changed GHSR expression. Indeed, we demonstrate here that chronic acyl-ghrelin administration following CSDS downregulates GHSR mRNA within the VTA. The VTA is potentially a key brain region through which the ghrelin system exerts its antidepressant-like effects. Not only do several studies suggest a critical role for a VTA to nucleus accumbens circuit in modulating behavioral responses to CSDS [59-61], previous work by our group has demonstrated that the exaggerated social avoidance phenotype of CSDS-exposed GHSR-null mice is rescued by catecholaminergic neuron-selective GHSR expression, including that in dopaminergic VTA neurons [15]. Notably, rats exposed to chronic immobilization stress also likely exhibit a reduction in GHSR expression, as suggested by a reduction in binding of labeled ghrelin within the basolateral amygdala [17]. Thus, perhaps the reduction in GHSR expression within the VTA or other regions such as the amygdala creates an environment of relative acyl-ghrelin resistance that hampers the capacity of the administered acyl-ghrelin or GHRP-2 to exert its antidepressant-like effects [17].

It is also plausible that the failed antidepressant-like efficacy of administered acyl-ghrelin or GHRP-2 relates to the administration protocols used. For instance, the single dose of acyl-ghrelin may have lacked efficacy in the context of CSDS, as other tested antidepressant agents require several weeks of administration to be effective, similar to their efficacy requirements in human subjects [31]. The chronic acyl-ghrelin administration protocol also results in high plasma levels of desacyl-ghrelin [33], which although not yet assessed for effects on depressive-like behavior, has been implicated in some studies as possessing actions related to anxiety-like behavior and actions opposite to those of acylghrelin $[4,5,18]$. That said, GHRP-2, which is more stable than acylghrelin, also was ineffective at improving the depressive-like behavior of CSDS-exposed mice [although neither did GHRP-2 increase body weight or food intake, unlike that observed previously with daily dosing [34]].

In summary, our data suggest that $\beta 1 A R$ signaling during CSDS is required for the rise in plasma acyl-ghrelin associated with chronic stress and protects against an exaggerated stress-induced depressive-like phenotype. While it is plausible that the raised plasma acyl-ghrelin mediates this behavioral effect of $\beta 1 A R$ signaling, such is not necessarily supported by the failure of administered acyl-ghrelin or administered GHRP-2 to limit CSDSassociated depressive-like behavior, albeit neither is it wholly refuted by those findings. A future investigation that could help clarify these issues might include co-administration of acyl-ghrelin alongside atenolol during CSDS to determine if replacement of acyl-ghrelin to normal stress-induced levels could reverse the behavioral phenotype of the atenolol-treated mice. Performing CSDS followed by the Social Interaction Test on mice with ghrelin cell-selective deletion of $\beta 1$ ARs [29] also would be instructive, providing further clues regarding the behavioral effects of the CSDS-induced rise in plasma acyl-ghrelin and the role of ghrelin cell $\beta 1 A R s$ in stress-induced acyl-ghrelin elevation. It would also be worthwhile to determine if changes to $\beta 1 A R$ signaling are responsible for the long-lasting elevations in plasma acyl-ghrelin reported in both rodent and human stress models. Additionally, studies incorporating pharmacologic or genetic manipulation of GHSR constitutive activity or GHSR heterodimerization with other $\mathrm{G}$ protein-coupled receptors could help clarify the behavioral effects of those components of the ghrelin system.

\section{FUNDING AND DISCLOSURE}

This work was supported by the NIH (R01 DK103884), the Diana and Richard C. Strauss Professorship in Biomedical Research, the Mr. and Mrs. Bruce G. Brookshire Professorship in Medicine, the Kent and Jodi Foster Distinguished Chair in Endocrinology, in Honor of Daniel Foster, M.D., and institutional funds from the University of Texas Southwestern Medical Center (to J.M.Z.)

\section{ACKNOWLEDGEMENTS}

We acknowledge Dr. Shari Birnbaum in the UTSW Behavioral Core for help with the behavioral tests.

\section{ADDITIONAL INFORMATION}

Supplementary Information accompanies this paper at (https://doi.org/10.1038/ s41386-019-0334-7).

Competing interests: The authors declare no competing interests.

Publisher's note: Springer Nature remains neutral with regard to jurisdictional claims in published maps and institutional affiliations.

\section{REFERENCES}

1. Sinha R. Role of addiction and stress neurobiology on food intake and obesity. Biol Psychol. 2018;131:5-13.

2. Patterson ZR, Abizaid A. Stress induced obesity: lessons from rodent models of stress. Front Neurosci. 2013;7:130.

3. Schellekens H, Dinan TG, Cryan JF. Taking two to tango: a role for ghrelin receptor heterodimerization in stress and reward. Front Neurosci. 2013;7:148.

4. Mani BK, Zigman JM. Ghrelin as a survival hormone. Trends Endocrinol Metab. 2017;28:843-54

5. Wittekind DA, Kluge M. Ghrelin in psychiatric disorders - a review. Psychoneuroendocrinology. 2015;52:176-94.

6. Meyer RM, Burgos-Robles A, Liu E, Correia SS, Goosens KA. A ghrelin-growth hormone axis drives stress-induced vulnerability to enhanced fear. Mol Psychiatry. 2014;19:1284-94.

7. Asakawa A, Inui A, Kaga T, Yuzuriha $H$, Nagata $T$, Fujimiya $M$, et al. A role of ghrelin in neuroendocrine and behavioral responses to stress in mice. Neuroendocrinology. 2001;74:143-7.

8. Li B, Xu Y, Pan D, Xiao Q, Gao Q, Chen X, et al. Effect of immobilization stress on the appetite and stomach ghrelin expression in maternal mice. Int J Clin Exp Pathol. 2015;8:15993-9.

9. Zheng J, Dobner A, Babygirija R, Ludwig K, Takahashi T. Effects of repeated restraint stress on gastric motility in rats. Am J Physiol Regul Integr Comp Physiol. 2009;296:R1358-65.

10. Ochi M, Tominaga K, Tanaka F, Tanigawa T, Shiba M, Watanabe T, et al. Effect of chronic stress on gastric emptying and plasma ghrelin levels in rats. Life Sci. 2008;82:862-8. 
11. Yamada C, Saegusa $Y$, Nahata M, Sadakane C, Hattori T, Takeda H. Influence of aging and gender differences on feeding behavior and ghrelin-related factors during social isolation in mice. PLoS ONE. 2015;10:e0140094.

12. Patterson $\mathrm{ZR}$, Khazall $\mathrm{R}$, Mackay $\mathrm{H}$, Anisman $\mathrm{H}$, Abizaid $\mathrm{A}$. Central ghrelin signaling mediates the metabolic response of $\mathrm{C} 57 \mathrm{BL} / 6$ male mice to chronic social defeat stress. Endocrinology. 2013;154:1080-91.

13. Yousufzai M, Harmatz ES, Shah M, Malik MO, Goosens KA. Ghrelin is a persistent biomarker for chronic stress exposure in adolescent rats and humans. Transl Psychiatry. 2018;8:74.

14. Lutter M, Sakata I, Osborne-Lawrence S, Rovinsky SA, Anderson JG, Jung S, et al. The orexigenic hormone ghrelin defends against depressive symptoms of chronic stress. Nat Neurosci. 2008;11:752.

15. Chuang JC, Perello M, Sakata I, Osborne-Lawrence S, Savitt JM, Lutter M, et al. Ghrelin mediates stress-induced food-reward behavior in mice. J Clin Invest. 2011;121:2684-92.

16. Kumar J, Chuang JC, Na ES, Kuperman A, Gillman AG, Mukherjee S, et al. Differential effects of chronic social stress and fluoxetine on meal patterns in mice. Appetite. 2013;64:81-88.

17. Harmatz ES, Stone L, Lim SH, Lee G, McGrath A, Gisabella B, et al. Central Ghrelin resistance permits the overconsolidation of fear memory. Biol Psychiatry. 2017;81:1003-13.

18. Spencer SJ, Emmerzaal TL, Kozicz T, Andrews ZB. Ghrelin's role in the hypothalamic-pituitary-adrenal axis stress response: implications for mood disorders. Biol Psychiatry. 2015;78:19-27.

19. Nahata M, Saegusa Y, Sadakane C, Yamada C, Nakagawa K, Okubo N, et al. Administration of exogenous acylated ghrelin or rikkunshito, an endogenous ghrelin enhancer, improves the decrease in postprandial gastric motility in an acute restraint stress mouse model. Neurogastroenterol Motil. 2014;26:821-31.

20. Morris LS, Voon V, Leggio L. Stress, motivation, and the gut-brain axis: a focus on the ghrelin system and alcohol use disorder. Alcohol Clin Exp Res. 2018;42:1378-9.

21. Nakashima K, Akiyoshi J, Hatano K, Hanada H, Tanaka Y, Tsuru J, et al. Ghrelin gene polymorphism is associated with depression, but not panic disorder. Psychiatr Genet. 2008;18:257.

22. Kluge $M$, Schussler $P$, Dresler $M$, Schmidt $D$, Yassouridis $A$, Uhr $M$, et al. Effects of ghrelin on psychopathology, sleep and secretion of cortisol and growth hormone in patients with major depression. J Psychiatr Res. 2011;45:421-6.

23. Carlini VP, Machado DG, Buteler F, Ghersi M, Ponzio MF, Martini AC, et al. Acute ghrelin administration reverses depressive-like behavior induced by bilateral olfactory bulbectomy in mice. Peptides. 2012;35:160-5.

24. Walker AK, Rivera PD, Wang Q, Chuang JC, Tran S, Osborne-Lawrence $S$, et al The P7C3 class of neuroprotective compounds exerts antidepressant efficacy in mice by increasing hippocampal neurogenesis. Mol Psychiatry. 2015; 20:500-8

25. Jansen AS, Nguyen XV, Karpitskiy V, Mettenleiter TC, Loewy AD. Central command neurons of the sympathetic nervous system: basis of the fight-or-flight response. Science. 1995;270:644-6.

26. Keeney AJ, Hogg S, Marsden CA. Alterations in core body temperature, locomotor activity, and corticosterone following acute and repeated social defeat of male NMRI mice. Physiol Behav. 2001;74:177-84.

27. Sgoifo A, Koolhaas J, De Boer S, Musso E, Stilli D, Buwalda B, et al. Social stress, autonomic neural activation, and cardiac activity in rats. Neurosci Biobehav Rev. 1999;23:915-23.

28. Mundinger TO, Cummings DE, Taborsky GJ Jr.. Direct stimulation of ghrelin secretion by sympathetic nerves. Endocrinology. 2006;147:2893-901.

29. Mani BK, Osborne-Lawrence S, Vijayaraghavan P, Hepler C, Zigman JM. beta1Adrenergic receptor deficiency in ghrelin-expressing cells causes hypoglycemia in susceptible individuals. J Clin Invest. 2016;126:3467-78.

30. Zhao TJ, Sakata I, Li RL, Liang G, Richardson JA, Brown MS, et al. Ghrelin secretion stimulated by beta\}1-adrenergic receptors in cultured ghrelinoma cells and in fasted mice. Proc Natl Acad Sci USA. 2010;107:15868-73.

31. Golden SA, Covington HE III, Berton O, Russo SJ. A standardized protocol for repeated social defeat stress in mice. Nat Protoc. 2011;6:1183-91.

32. Chuang JC, Krishnan V, Yu HG, Mason B, Cui H, Wilkinson MB, et al. A beta3adrenergic-leptin-melanocortin circuit regulates behavioral and metabolic changes induced by chronic stress. Biol Psychiatry. 2010;67:1075-82.

33. Chuang JC, Sakata I, Kohno D, Perello M, Osborne-Lawrence S, Repa JJ, et al Ghrelin directly stimulates glucagon secretion from pancreatic alpha-cells. Mol Endocrinol. 2011;25:1600-11.

34. Tschop M, Statnick MA, Suter TM, Heiman ML. GH-releasing peptide-2 increases fat mass in mice lacking NPY: indication for a crucial mediating role of hypothalamic agouti-related protein. Endocrinology. 2002;143:558-68.

35. Hoffmann C, Leitz MR, Oberdorf-Maass S, Lohse MJ, Klotz KN. Comparative pharmacology of human beta-adrenergic receptor subtypes--characterization of stably transfected receptors in $\mathrm{CHO}$ cells. Naunyn Schmiede Arch Pharmacol. 2004;369:151-9.

36. McDevitt DG. Pharmacologic aspects of cardioselectivity in a beta-blocking drug Am J Cardiol. 1987;59:10F-2.

37. Westerlund A. Central nervous system side-effects with hydrophilic and lipophilic beta-blockers. Eur J Clin Pharmacol. 1985;28:73-6.

38. de la Cour CD, Norlen $P$, Hakanson R. Secretion of ghrelin from rat stomach ghrelin cells in response to local microinfusion of candidate messenger compounds: a microdialysis study. Regul Pept. 2007;143:118-26.

39. Sakata I, Park WM, Walker AK, Piper PK, Chuang JC, Osborne-Lawrence S, et al. Glucose-mediated control of ghrelin release from primary cultures of gastric mucosal cells. Am J Physiol Endocrinol Metab. 2012;302:E1300-10.

40. Gagnon J, Anini Y. Insulin and norepinephrine regulate ghrelin secretion from a rat primary stomach cell culture. Endocrinology. 2012;153:3646-56.

41. Iwakura $\mathrm{H}$, Ariyasu $\mathrm{H}$, Hosoda $\mathrm{H}$, Yamada G, Hosoda $\mathrm{K}$, Nakao K, et al. Oxytocin and dopamine stimulate ghrelin secretion by the ghrelin-producing cell line, MGN3-1 in vitro. Endocrinology. 2011;152:2619-25.

42. Mani BK, Chuang JC, Kjalarsdottir L, Sakata I, Walker AK, Kuperman A, et al. Role of calcium and EPAC in norepinephrine-induced ghrelin secretion. Endocrinology. 2014;155:98-107.

43. Engelstoft MS, Park WM, Sakata I, Kristensen LV, Husted AS, Osborne-Lawrence S, et al. Seven transmembrane $G$ protein-coupled receptor repertoire of gastric ghrelin cells. Mol Metab. 2013;2:376-92.

44. Koyama H, Iwakura H, Dote K, Bando M, Hosoda H, Ariyasu H, et al. Comprehensive profiling of GPCR expression in ghrelin-producing cells. Endocrinology. 2016;157:692-704.

45. Ariyasu H, Takaya K, Tagami T, Ogawa Y, Hosoda K, Akamizu T, et al. Stomach is a major source of circulating ghrelin, and feeding state determines plasma ghrelin-like immunoreactivity levels in humans. J Clin Endocrinol Metab. 2001; 86:4753-8.

46. Homan P, Grob S, Milos G, Schnyder U, Hasler G. Reduction in total plasma ghrelin levels following catecholamine depletion: relation to bulimic and depressive symptoms. Psychoneuroendocrinology. 2013;38:1545-52.

47. Rosen RC, Kostis JB. Biobehavioral sequellae associated with adrenergic-inhibiting antihypertensive agents: a critical review. Health Psychol. 1985;4:579-604.

48. Stemmelin J, Cohen C, Terranova JP, Lopez-Grancha M, Pichat P, Bergis O, et al Stimulation of the beta3-Adrenoceptor as a novel treatment strategy for anxiety and depressive disorders. Neuropsychopharmacology. 2008;33:574-87.

49. Iwai T, Hayashi Y, Narita S, Kasuya Y, Jin K, Tsugane M, et al. Antidepressant-like effects of glucagon-like peptide- 2 in mice occur via monoamine pathways. Behav Brain Res. 2009;204:235-40.

50. Shahzad N, Ahmad J, Khan W, Al-Ghamdi SS, Ain MR, Ibrahim IA, et al. Interactions of atenolol with alprazolam/escitalopram on anxiety, depression and oxidative stress. Pharmacol Biochem Behav. 2014;117:79-84.

51. Tanaka M, Telegdy G. Involvement of adrenergic and serotonergic receptors in antidepressant-like effect of urocortin 3 in a modified forced swimming test in mice. Brain Res Bull. 2008;77:301-5.

52. Hymel KA, Eans SO, K LS, Gomes SM, Lukowsky AL, Medina JM, et al. Stressinduced increases in depression-like and cocaine place-conditioned behaviors are reversed by disruption of memories during reconsolidation. Behav Pharmacol. 2014;25:599-608.

53. Verbeek DE, van Riezen J, de Boer RA, van Melle JP, de Jonge P. A review on the putative association between beta-blockers and depression. Heart Fail Clin. 2011;7:89-99.

54. Ko DT, Hebert PR, Coffey CS, Sedrakyan A, Curtis JP, Krumholz HM. Beta-blocker therapy and symptoms of depression, fatigue, and sexual dysfunction. JAMA 2002:288:351-7.

55. Ried LD, McFarland BH, Johnson RE, Brody KK. Beta-blockers and depression: the more the murkier? Ann Pharmacother. 1998;32:699-708.

56. Terao T. Beta-blocker therapy and depression. JAMA. 2002;288:1845.

57. Ried LD, Tueth MJ, Taylor MD, Sauer BC, Lopez LM, Pepine CJ. Depressive symptoms in coronary artery disease patients after hypertension treatment. Ann Pharmacother. 2006;40:597-604.

58. Huang HJ, Zhu XC, Han QQ, Wang YL, Yue N, Wang J, et al. Ghrelin alleviates anxiety- and depression-like behaviors induced by chronic unpredictable mild stress in rodents. Behav Brain Res. 2017:326:33-43.

59. Nestler EJ, Carlezon WA Jr.. The mesolimbic dopamine reward circuit in depression. Biol Psychiatry. 2006;59:1151-9.

60. Krishnan V, Han MH, Graham DL, Berton O, Renthal W, Russo SJ, et al. Molecular adaptations underlying susceptibility and resistance to social defeat in brain reward regions. Cell. 2007;131:391-404.

61. Chaudhury D, Walsh JJ, Friedman AK, Juarez B, Ku SM, Koo JW, et al. Rapid regulation of depression-related behaviours by control of midbrain dopamine neurons. Nature. 2013;493:532-6. 